\title{
Preparation and Characterization of Irinotecan Loaded Cross-Linked Bovine Serum Albumin Beads for Liver Cancer Chemoembolization Therapy
}

\author{
Jin Yan, ${ }^{1}$ Fei Wang, ${ }^{1}$ Jun Chen, ${ }^{2}$ Tao Liu, ${ }^{3}$ and Tao Zhang ${ }^{1}$ \\ ${ }^{1}$ College of Engineering and Applied Sciences, Nanjing University, Nanjing 210093, China \\ ${ }^{2}$ Department of Radiology, Jiangsu Cancer Hospital and Cancer Hospital of Nanjing Medical University, Nanjing 210009, China \\ ${ }^{3}$ Jiangsu Provincial Key Laboratory for Interventional Medical Devices, Huaiyin Institute of Technology, Huaian 223003, China \\ Correspondence should be addressed to Tao Zhang; ztnj@nju.edu.cn
}

Received 3 January 2016; Revised 18 March 2016; Accepted 21 March 2016

Academic Editor: Cornelia Vasile

Copyright (C) 2016 Jin Yan et al. This is an open access article distributed under the Creative Commons Attribution License, which permits unrestricted use, distribution, and reproduction in any medium, provided the original work is properly cited.

\begin{abstract}
In this paper, a novel temporary embolization agent for transarterial chemoembolization of liver cancer was developed and tested. The Irinotecan loaded bovine serum albumin (BSA) beads were tried to be used as embolic agent of liver cancer therapy. BSA beads were prepared by a water-in-oil emulsion solvent diffusion method in soya oil and Span 85 was used as the emulsifier. The obtained BSA beads were able to swell 2.37-fold comparing to dried beads. Depending on the equilibrium swelling process, the Irinotecan was loaded with $9.8 \%$ total drug concentration and tested. In vitro drug release studies showed that a burst release of Irinotecan was achieved. Eventually BSA beads were completely degraded in a few weeks. CCK-8 assay demonstrated that BSA beads showed no cytotoxicity against human umbilical vein endothelial cells, and the Irinotecan loaded BSA beads showed comparable cytotoxicity against Hep G2, a human liver carcinoma cell line, as the traditional Irinotecan. In a rabbit model, it was found that BSA beads can successfully be transferred to liver and provide occlusion of small arteries. The present investigation suggested that the BSA beads are promising drug carriers and can potentially be used as temporary embolization agents in interventional oncology.
\end{abstract}

\section{Introduction}

Nowadays, most liver cancers are diagnosed at mediate or advanced stages and few effective treatment methods are available [1]. Transarterial chemoembolization (TACE) is a catheter based interventional oncologic therapy that serves as one of the most promising therapies for unresectable primary hepatocellular carcinoma (HCC) and metastatic liver cancers $[2,3]$. The basic form of conventional TACE involves a twostep process starting with the intra-arterial administration of chemotherapeutic agents mixed with contrast agents into the tumor-feeding artery via an intra-arterially inserted catheter and followed by selective embolization of the tumor-feeding artery. A series of novel and simplified TACE therapies have been developed in the last decades, such as lipiodol $[4,5]$, gelatin sponges [6, 7], and polyvinyl alcohol (PVA) particles [8]. More recently, these therapies involve the use of drug eluting beads (DEBs) [9], in which the drug loaded carriers are injected directly into arteries and then the drug carriers will carry the therapeutic agents to sites of tumor, followed by the spontaneous elution of drugs from the carriers into cancer tissues. Typical methods of this regard include DC Beads [10], HepaSphere [11], and gelatin microspheres [12].

Most of the current DEBs are prepared with nondegradable polymers, but, in theory, biodegradable agents probably are more favorable for the following reasons $[12,13]$ : (1) the agents only work during treatment; therefore, the embolism syndromes can be avoided, (2) bioabsorbable microspheres provide a possibility of repeated embolization after recanalization of vessels in the subsequent chemotherapies, and (3) by taking cell cycles into consideration, repeated therapy probably will be more effective for the same tumor bed for the reason that repeat therapy can kill cancer cells completely. Some of the mostly used biodegradable agents are albumin $[14,15]$, gelatin $[7,16]$, starch [17], chitosan [1821], and so forth. What is worth mentioning is that albumin 
is a promising material and has been extensively investigated as a biocompatible carrier partially thanks to its biodegradability, nontoxicity, and nonimmunogenicity and partially because a variety of drugs can be incorporated onto the material in a relatively nonspecific fashion [22-24]. Among albumins, bovine serum albumin (BSA) is a serum albumin protein derived from cows and can incorporate a variety of drugs following a relatively nonspecific protocol [23]. In addition, due to the large content of charged amino acids, BSA beads can allow electrostatic adsorption of both positively and negatively charged molecules without the requirements of other modifications. In addition, the functional groups, such as carboxylic and amino groups, in the BSA molecules can facilitate the noncovalent attachment of drug molecules [25].

In the previous reports [26], theophylline-loaded BSA microspheres were prepared by an emulsion polymerization method using glutaraldehyde as the cross-linking agent and the results indicated that the BSA microspheres were able to control theophylline release slowly. In this paper, BSA beads were prepared by the emulsion method and the physical, chemical, and biological properties of the products were studied and reported. Furthermore, a widely used antitumor drug, irinotecan $[27,28]$, was incorporated to form the drug eluting beads. The in vitro drug release, degradation, and cytotoxicity of the beads are reported. In addition, the in vivo embolization using rabbit as a model has also been investigated.

\section{Experimental}

2.1. Materials. Bovine serum albumin (BSA, 96\%) was supplied by Nanjing Duly Biotech Co., Ltd. Glutaraldehyde (GA, $50 \%$ in water), Span 85 , acetone, $n$-hexane, and other chemicals were all purchased from Sinopharm Chemical Reagent Co. (Shanghai, China) and used as received. Dulbecco's modified Eagle's medium (DMEM, with $4500 \mathrm{mg} / \mathrm{L}$ glucose and L-glutamine) was purchased from Thermo Fisher Scientific China Branch (Beijing, China). Fetal bovine serum (FBS) was supplied by Hangzhou Sijiqing Biological Engineering Materials Co., Ltd. (Hangzhou, China), which was heat inactivated for $30 \mathrm{~min}$ at $56^{\circ} \mathrm{C}$ and then stored at $20^{\circ} \mathrm{C}$ before use. Cell Counting Kit-8 (CCK-8) was obtained from Dojindo Molecular Technologies, Inc. (Shanghai, China).

\subsection{Preparation of BSA Beads and Irinotecan Loaded BSA} Beads. BSA beads were prepared by a water-in-oil (w/o) emulsion solvent diffusion method. In the preparation, $6 \mathrm{~mL}$ $20 \%$ (wt/v) BSA water solution was added dropwise to $60 \mathrm{~mL}$ soya oil containing $2 \%(\mathrm{v} / \mathrm{v})$ Span 85 with stirring speed of $900 \mathrm{rpm}$ at $60^{\circ} \mathrm{C}$. After being stirred for $1 \mathrm{~h}$ to obtain a stable emulsion, $400 \mu \mathrm{L}$ glutaraldehyde (10\%) was added with continued stirring for more than $2 \mathrm{~h}$ to cross-link the beads. The soya oil was discarded and the crude beads were rinsed in $n$-hexane and acetone subsequently for several times until the soya oil was washed out thoroughly. The cross-linked BSA beads were then vacuum-dried at room temperature.
In order to load irinotecan into BSA beads, $200 \mu \mathrm{L}$ irinotecan solution $(50 \mathrm{mg} / \mathrm{mL})$ was mixed with $50 \mathrm{mg}$ dried BSA beads. After being completely swelled, the beads were dried with lyophilization at $-65^{\circ} \mathrm{C}$ for $72 \mathrm{~h}$ and the actual amount of irinotecan enwrapped in BSA beads was determined. In detail, $10 \mathrm{mg}$ irinotecan loaded dried BSA beads were ground with an agate mortar and then dispersed in $10 \mathrm{~mL}$ phosphate buffered saline (PBS, pH 7.4, $0.1 \mathrm{~mol} / \mathrm{L}$ ) and stirred for $1 \mathrm{~h}$. Then, the suspended fragments were removed by centrifugation. The concentration of irinotecan in supernatant was measured with a Mapada UV1800PC ultraviolet-visible spectrophotometer (Shanghai, China) at $369 \mathrm{~nm}$ wavelength. The drug loading content was calculated as the weight percent of irinotecan in BSA beads and the drug encapsulation efficiency was calculated as the weight percent of enwrapped irinotecan to original fed drugs.

2.3. Morphological Investigations. The appearances of the beads were monitored with an Axio Scope Al fluorescence microscope (Zeiss, German) and the sizes of beads were determined by measuring the diameters of the beads (at least 200) in the microscope photographs both before and after swelling. The swelling ratios of beads were calculated as $\left(D_{t}-D_{0}\right) / D_{0} \times 100 \%$, where $D_{0}$ represented the diameter of beads before swelling while $D_{t}$ represented the diameter after swelling. The samples with gold coating (approx. $40 \mathrm{~nm}$ thickness) were also observed under a SEM (S-3400N II, Hitachi, Japan) at an acceleration voltage of $20 \mathrm{kV}$ in order to investigate the micromorphology of BSA beads.

2.4. Chemical Structural Determinations. Fourier transform infrared spectra (FTIR) of samples were recorded with a PE GX FTIR spectrometer (Perkin-Elmer, USA) at room temperature on $\mathrm{KBr}$ pellets with sample concentrations of $1 \%$ from 4000 to $400 \mathrm{~cm}^{-1}$ with a resolution of $2 \mathrm{~cm}^{-1}$. Wide angle X-ray diffraction (XRD) measurements were performed on a Rigaku ULTIMA-3 setup with Mar 345 image plate as detector and $\mathrm{CuK} \alpha$ was used as the source (wavelength $D=0.1542 \mathrm{~nm}$ ). The recorded region of $2 \theta$ was from $10^{\circ}$ to $40^{\circ}$ with scanning speed $2^{\circ} \cdot \mathrm{min}^{-1}$.

2.5. In Vitro Degradation Determination of BSA Beads. In order to evaluate the degradation of cross-linked BSA beads, all $25 \mathrm{mg}$ dried beads were put in sealed vial with $4 \mathrm{~mL}$ Tyrosine PBS solution $(50 \mu \mathrm{g} / \mathrm{mL})$ and then placed in a constant temperature shaker heated to $37 \pm 1^{\circ} \mathrm{C}$. In order to guarantee the bioactivity of Tyrosine, half of the solution was changed every two days. At scheduled time points, the beads were filtered from the solution, washed in pure water and ethanol for several times, and finally vacuum-dried and weighed. The relative degradation ratio was calculated by $\left(m_{0}-m_{t}\right) / m_{0} \times 100 \%$, where $m_{0}$ is the original mass of beads and $m_{t}$ represented the remaining mass after degradation.

2.6. In Vitro Drug Release from Cross-Linked BSA Beads. All $10 \mathrm{mg}$ irinotecan loaded BSA beads were suspended in $2 \mathrm{~mL}$ PBS $(0.01 \mathrm{~mol} / \mathrm{L}, \mathrm{pH}$ 7.4) in dialysis bag (molecular weight cut-off $3000 \mathrm{Da}$ ) and then the bags were immersed 
into $100 \mathrm{~mL} 0.01 \mathrm{M}$ PBS with $\mathrm{pH} 7.4$ which was used as release medium and was kept at $37^{\circ} \mathrm{C}$. At every presupposed time point, $3 \mathrm{~mL}$ release medium was taken out and replaced with $3 \mathrm{~mL}$ fresh PBS. The samples were analyzed by UV-visible spectrometer at $369 \mathrm{~nm}$ to determine the concentration of irinotecan.

2.7. In Vitro Cytotoxicity Studies. The cytotoxicity of BSA beads against human umbilical vein endothelial cells (HUVEC) and irinotecan loaded BSA beads against human liver carcinoma cell line (Hep G2) was determined by CCK-8 assay. In order to evaluate the potential cytotoxicity of BSA beads, cells were seeded in 96-well plates with DMEM supplemented with $10 \%$ calf serum and incubated at $37^{\circ} \mathrm{C}$ with $5 \% \mathrm{CO}_{2}$ for $24 \mathrm{~h}$. Cells were then exposed to various doses of BSA beads for $24 \mathrm{~h}$, followed by incubation with $20 \mu \mathrm{L}$ of CCK-8 ( $5 \mathrm{mg} / \mathrm{mL}$ in culture medium) for $1 \mathrm{~h}$ at $37^{\circ} \mathrm{C}$. The absorbance of the supernatant at $450 \mathrm{~nm}$ was recorded with a Rayto RT-6000 microplate reader. Every experiment was performed at least in sextuplicate. The Hep G2 cells for antitumor activity studies were exposed to various doses of irinotecan and irinotecan loaded BSA beads for $24 \mathrm{~h}$ and dealt with in the same way like the abovementioned way.

2.8. In Vivo Rabbit Model. Animal experiments were designed to preevaluate the feasibility of embolization in a healthy rabbit. Animal experimental protocols were approved by our Institutional Animal Care and Use Committee (IACUC) of College of Engineering and Applied Sciences, Nanjing University. A New Zealand white rabbit weighing 4-5 Kg was catheterized with X-ray fluoroscopic guidance by using a C-arm unit (Philips Allura Xper FD20). The right common femoral artery was surgically exposed. A 2.7-F catheter (MC-PE27131, Terumo, Tokyo, Japan) was inserted and advanced over a 0.014-inch-diameter guidewire into the targeted hepatic artery. BSA beads dispersed in saline were delivered slowly through the catheter until the total occlusion of the artery was reached. Conventional digital subtraction angiography of hepatic artery was performed before and after the embolization. After the procedure, the animal was euthanized by the injection of $10 \mathrm{~mL}$ air through the auricular vein under sedation.

\section{Results and Discussion}

3.1. Morphologies of BSA Beads and Irinotecan Loaded BSA Beads. Figure 1 shows the morphology of cross-linked BSA beads and irinotecan loaded BSA beads. Under bright field optical microscope as shown in Figure 1(a), the beads appear as entirely spherical with size ranging from 100 to 200 micrometers. Under dark field fluorescence microscope (Figure 1(b)), the beads show green fluorescence. After being lyophilized, the cross-linked BSA beads shrank to 20 to 100 micrometers as observed by SEM but still kept spherical as shown in Figure 1(c). With magnification of 10000x under SEM, the beads showed smooth surface with slight fluctuation as in Figure 1(d). Then, the cross-linked BSA beads were incorporated with irinotecan in waterish condition, freeze-dried again, and investigated with SEM; it was found that the beads kept stable with almost no change, even in surface micromorphology (Figure $1(\mathrm{e})$ ). The evaluation of size distribution of BSA beads as illustrated in Figure 1(f) shows the size distribution of the cross-linked BSA beads before and after swelling. It was found that $80 \%$ of the fully swelled beads were in size range from 120 to $180 \mu \mathrm{m}$ with average particle size of $150 \mu \mathrm{m}$ and only $20 \%$ were oversized. While the dried BSA beads had an average size of $60 \mu \mathrm{m}$ and swelled 2.37-fold the original size, the swelling ratio for the BSA beads is presented as $137 \%$.

3.2. FTIR and XRD Characterization of BSA Beads. The molecular structure of BSA consists of amino acids, which can react with glutaraldehyde to form a series of complex cross-linked structures, including the formation of Schiff base structure [29]. FTIR spectroscopy is an effective technique to study the secondary structure of proteins before and after cross-linking and it can be applied to analyze the chemical structure transformations of BSA molecule. Figure 2(a) shows the FTIR absorption spectra of pristine BSA and BSA beads. The FTIR absorption peaks of pristine BSA at 3299, 3062,1656 , and $1535 \mathrm{~cm}^{-1}$ are assigned to the stretching vibration of $\mathrm{O}-\mathrm{H}$, amide $\mathrm{A}$ (mainly $\mathrm{N}-\mathrm{H}$ stretching vibration), amide I, and amide II bands, respectively. The amide I band is the characteristic band of the $\mathrm{C}=\mathrm{O}$ stretching vibrations, while amide II is in the phase combination of $\mathrm{N}-\mathrm{H}$ in plane bending and $\mathrm{C}=\mathrm{N}$ stretching. Comparing with pristine $\mathrm{BSA}$, the reduced intensity of N-H bend in BSA cross-linked BSA beads was due to the amide bonding of amine groups of BSA with GA. The FTIR peak of BSA beads at $1671 \mathrm{~cm}^{-1}$ shows the formation of Schiff base structure. Because the reactions between BSA and GA were intentionally controlled to prepare the light cross-linked beads, the FTIR peak of Schiff base structure in BSA beads was smaller but still it could be observed.

The WAXRD spectra of pristine BSA and BSA beads were shown in Figure 2(b). The peak intensity of BSA beads was a little stronger than that of BSA, and the peak shape was sharper. Both of them had low and broad peaks at around $2 \theta$ of $20^{\circ}$. Since the BSA beads consisted of inhomogeneous BSA molecules, therefore the WAXRD result showed nearly an amorphous state. After cross-linking, aggregation structure of BSA molecules in BSA beads showed almost no changes and still showed an amorphous state. To summarize the results of FTIR and WAXRD, the BSA was successfully crosslinked by GA and the properties of BSA in beads did not change significantly.

3.3. Irinotecan Loading and Release Pattern of BSA Beads. The irinotecan loading and encapsulation of three different batches of BSA beads were assessed by UV spectrophotometry. The results showed that irinotecan was loaded in cross-linked BSA beads at $9.8 \%$ mass percentage, achieving a loading efficiency of 58.7\%. In Figure 3(a), FTIR curves of pure irinotecan, BSA beads incorporated with irinotecan, and BSA beads without irinotecan were shown. From the spectrum of irinotecan incorporated BSA beads, the absorption 


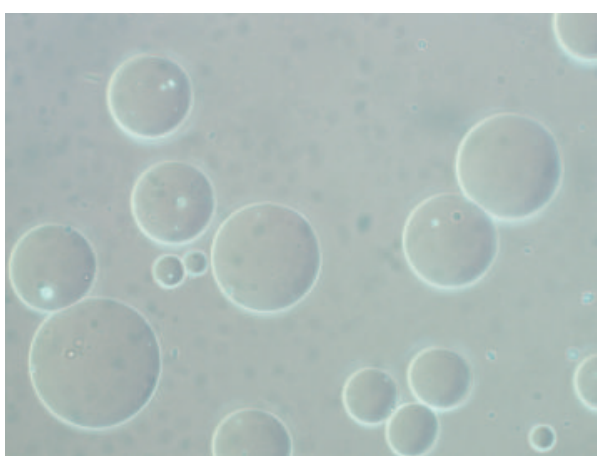

(a)

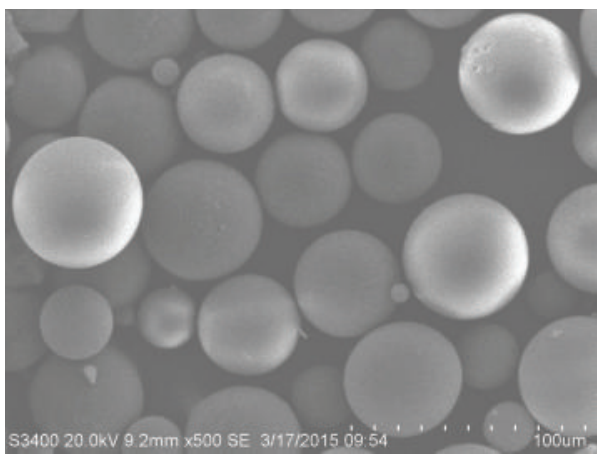

(c)

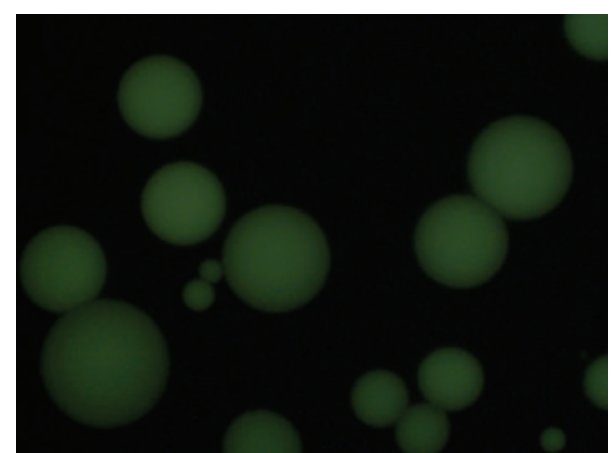

(b)

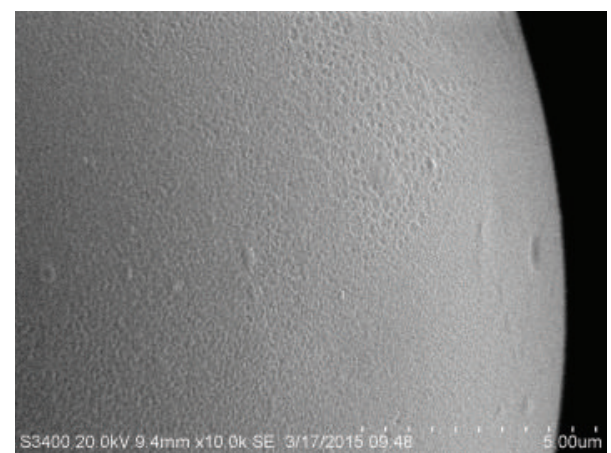

(d)

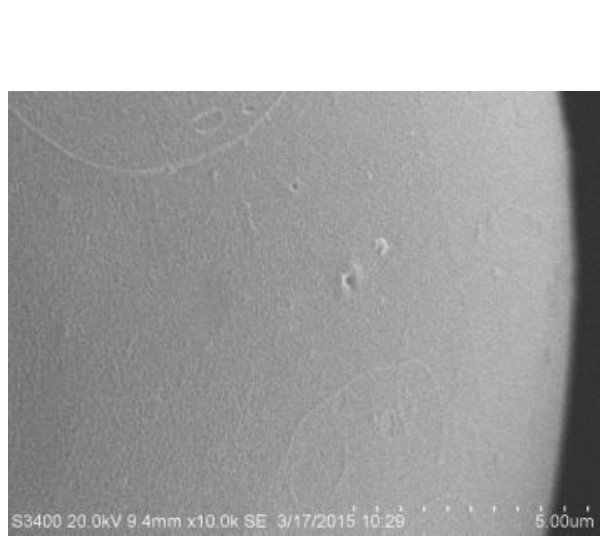

100

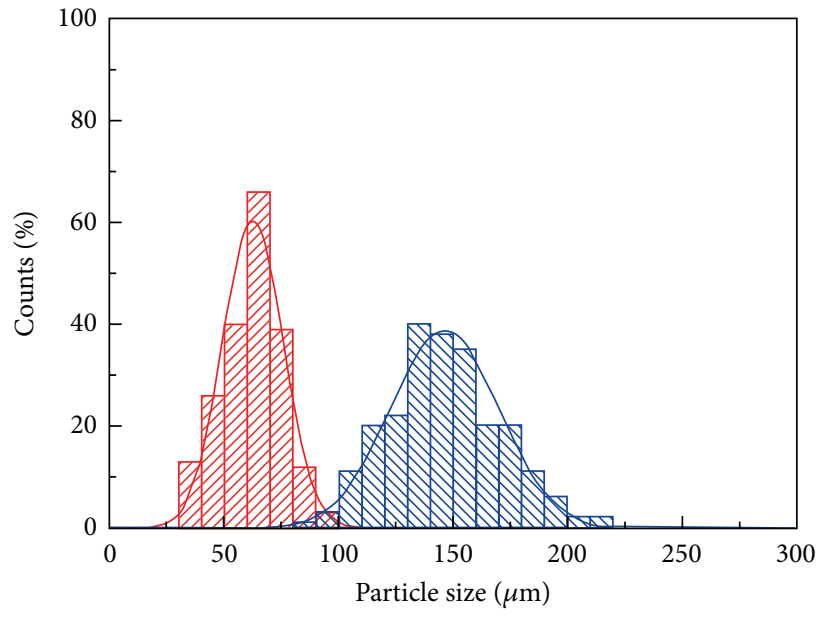

Z7 Dried BSA beads

MV Swelled beads

(f)

FIgure 1: Morphologies and size distributions of BSA beads and irinotecan loaded BSA beads. (a) Water-swelled cross-linked BSA beads with magnification of 200x under bright field optical microscope; (b) the same beads as (a) under dark field fluorescence microscope; (c) SEM images of dried cross-linked BSA beads with magnification of 500x; (d) surface morphology image of dried cross-linked BSA beads with magnification of 10000x under SEM; (e) surface morphology image of irinotecan loaded dried BSA beads with magnification of 10000x under SEM; and (f) size distribution of dried BSA beads and swelled BSA beads.

peaks of irinotecan and BSA were observed, indicating the successful loading of irinotecan into BSA beads. When the irinotecan loaded BSA beads were immersed into release medium, irinotecan was dissolved and released from the beads following the release curve as shown in Figure 3(b).
The release of irinotecan followed a swelling-controlled release mechanism and burst release was observed: $80.6 \%$ of irinotecan was released in the first 5 hours. After that, steady release took place and continued for 40 hours to finally achieve the release of $88.4 \%$ of the drugs. 


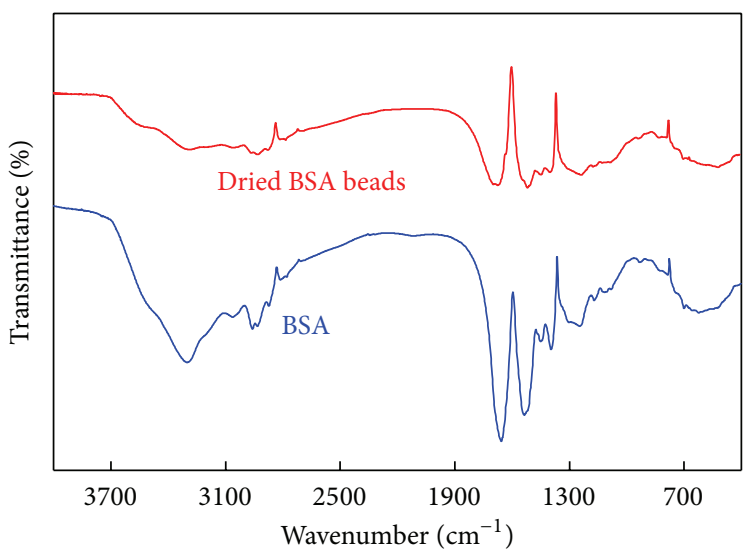

(a)

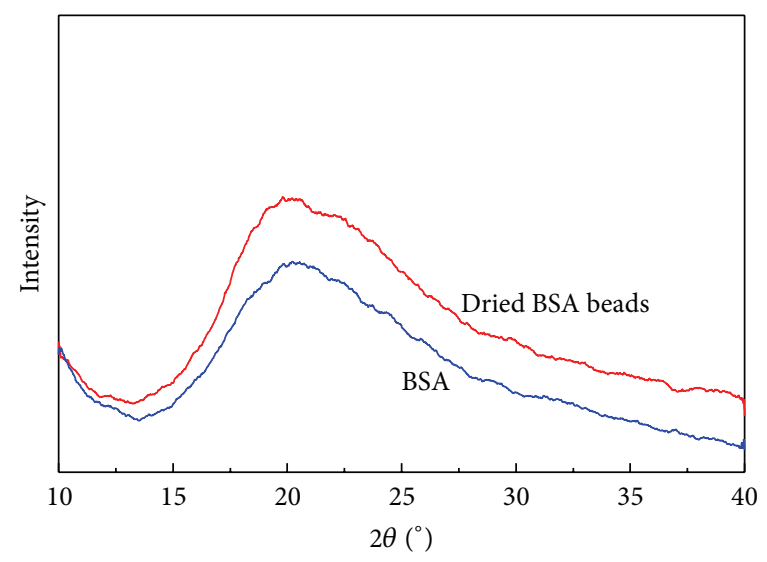

(b)

FIGURE 2: The FTIR spectra (a) and WAXRD curves (b) of BSA and dried cross-linked BSA beads.

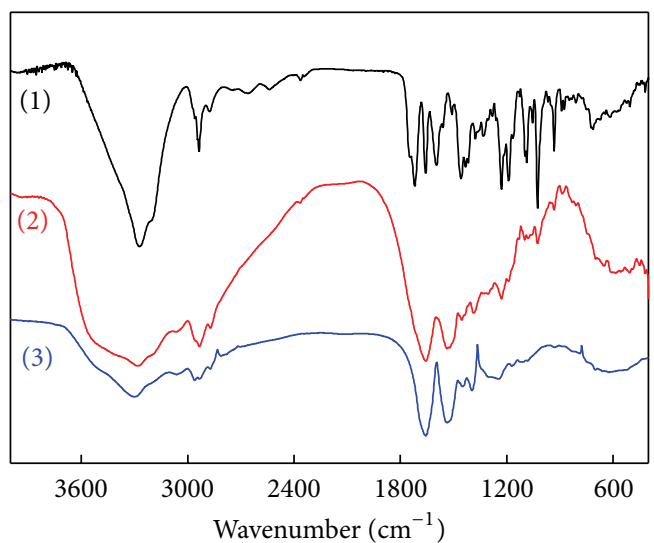

(1) Irinotecan

(2) Irinotecan in BSA beads

(3) BSA beads

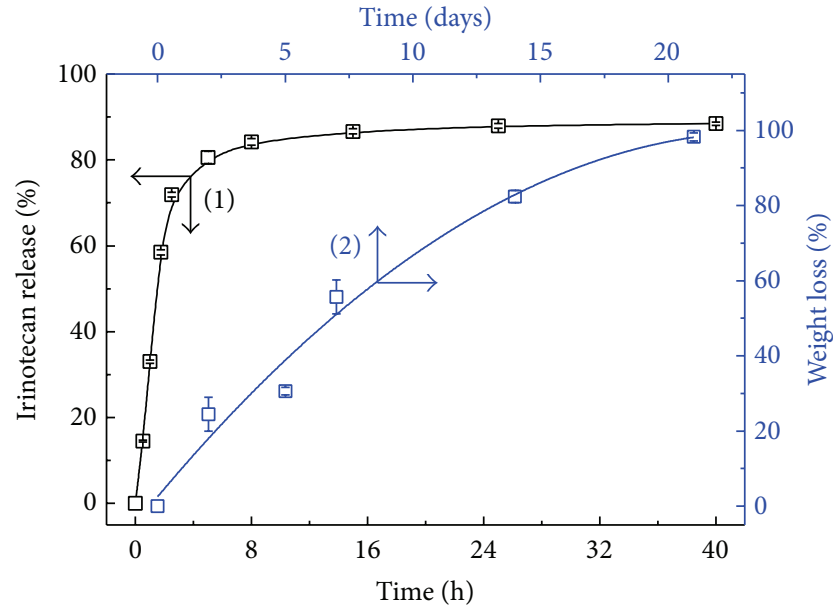

(1) Drug delivery curve of irinotecan

(2) In vitro degradation of BSA beads

(a)

(b)

FIGURE 3: (a) FTIR characterizations of irinotecan, BSA beads, and irinotecan loaded BSA beads; (b) irinotecan release curve from crosslinked BSA beads and the weight loss of BSA loaded BSA beads caused by degradation of BSA.

In our system, irinotecan was absorbed into cross-linked BSA beads and then was encapsulated in the beads after lyophilization. When the beads were immersed in the water again, irinotecan dissolved and diffused from the beads to the release medium. Actually, the release of irinotecan is governed by diffusion and it is influenced by the swelling characteristics of the materials which is dependent on the cross-linking degree of BSA. Comparing to the non-crosslinked system, the cross-linking in BSA beads reduced the mobility of BSA chain segments and compressed the space among the BSA molecules, which was expressed as the decrease of diffusion coefficient and resulted as the slow release of irinotecan from the cross-linked BSA beads.

As a natural protein, BSA is degradable, especially in an enzyme filled environment. Thus, the degradation profile was evaluated in Tyrosine PBS solution and the result was shown in Figure 3(b). The beads were almost completely degraded within 4 weeks, with the structure changing from sphere to floccule finally becoming solution. The time gap between the irinotecan release and beads degradation also demonstrated that the drug release is not controlled by the degradation but followed a swelling-controlled release mechanism. The drug release and degradation process of irinotecan beads can serve the purpose of both initial drug feeding to the tumor, followed by embolism of the tumor, and then the reopen of the embolized artery by beads being degraded.

3.4. In Vitro Cytotoxicity Study of BSA Beads. The biocompatibility of cross-linked BSA beads and irinotecan loaded BSA beads was investigated by CCK- 8 assay. To test the potential cytotoxicity of BSA beads, HUVEC were treated 


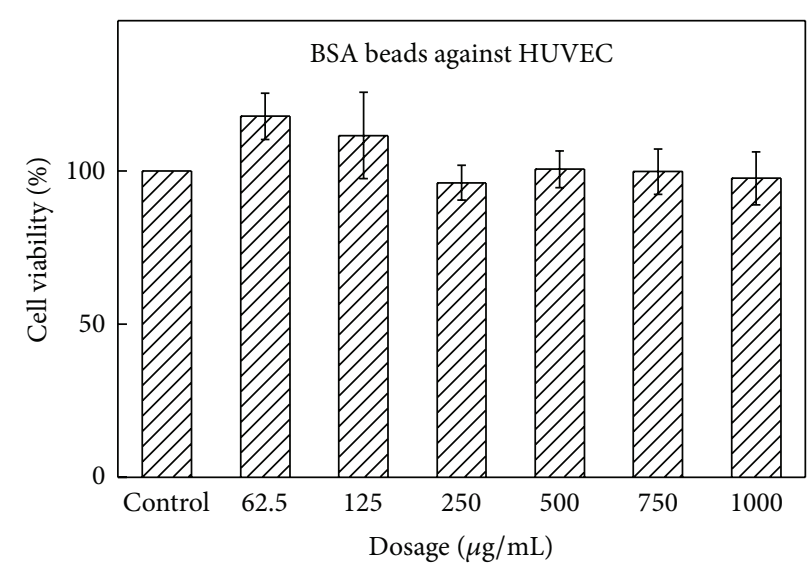

(a)

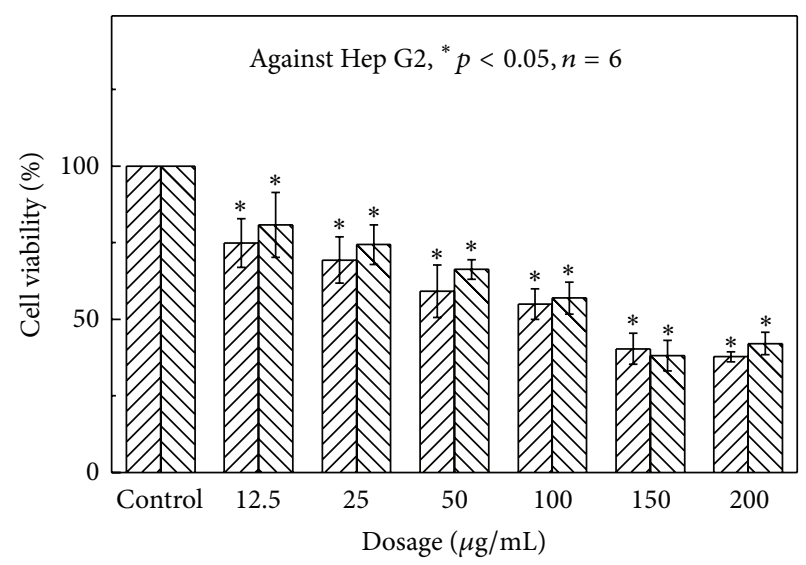

WIIS Irinotecan

Irinotecan loaded BSA beads

FIGURE 4: Cytotoxicity of BSA beads against HUVEC (a) and irinotecan loaded BSA beads against Hep G2 (b).

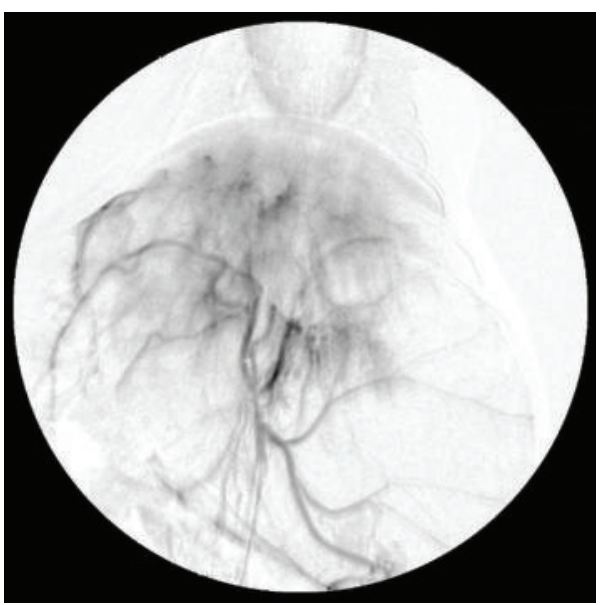

(a)

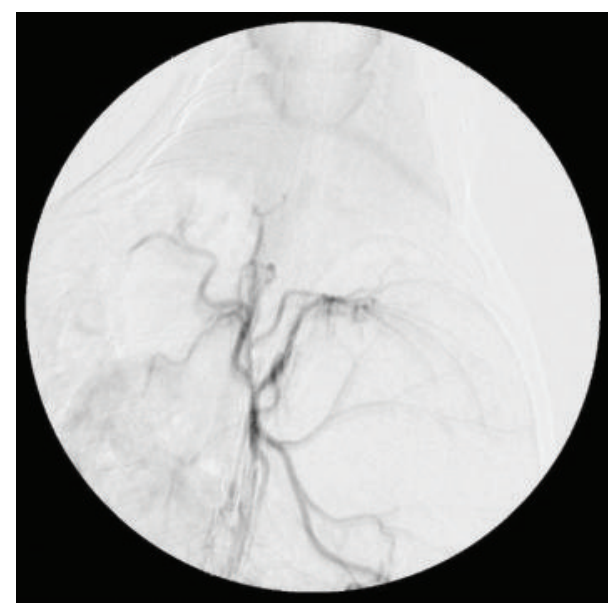

(b)

FIGURE 5: Angiograms of rabbit liver obtained before (a) and after (b) embolization by cross-linked BSA beads.

with BSA beads at various concentrations for $24 \mathrm{~h}$. As shown in Figure 4(a), BSA beads did not show any cytotoxicity against HUVEC even at the dosage of $1 \mathrm{mg} / \mathrm{mL}$; the cells grew well in all the concentrations of BSA beads comparing to the control group. Moreover, the cellular viability was significantly improved by incubating with the BSA beads at a concentration of $62.5 \mu \mathrm{g} / \mathrm{mL}$ for $24 \mathrm{~h}$. We speculate that the amino acids or peptides yielded from the degradation of BSA beads are beneficial to the cell proliferation. When the cells were cocultured with higher dosage of BSA beads, the growth area of cells became smaller because the cells preferred not to grow on the surface of the beads, which was observed under the microscope.

Figure 4(b) shows the in vitro tumor cell killing effects of free irinotecan and irinotecan loaded BSA beads against Hep G2. The cell viability of Hep-G2 at $200 \mu \mathrm{g} / \mathrm{mL}$ equivalent irinotecan dosage for $24 \mathrm{~h}$ was $37.8 \%$ and $42.1 \%$ for free irinotecan and irinotecan loaded BSA beads, respectively.
In the investigated dosage range, most of the cell viability of irinotecan loaded BSA beads group was slightly higher than that of free irinotecan group but was not of statistically significant difference. This phenomenon may be due to the positive impact of BSA itself, which resulted from the BAS beads decomposition. And the observation is consistent with the results from Figure 4(a). In summary, the irinotecan loaded BSA beads group shows similar cytotoxicity to free irinotecan against Hep G2. More importantly, the incorporation of irinotecan in BSA beads did not reduce its effect against tumor cells.

3.5. In Vivo Embolization Studies with BSA Beads. As an embolization agent for tumor-feeding arteries, the crosslinked BSA beads were evaluated in a healthy rabbit. Figure 5 shows the pre- and postembolization comparison of the rabbit hepatic arteries. The hepatic arteries and tumor 
staining before embolization were clearly observable as shown in Figure 5(a). After embolization, these arteries were no longer visible due to the vascular occlusion by the BSA beads (Figure 5(b)). A follow-up angiogram performed 15 min after embolization confirmed the persistent occlusion of the hepatic arteries, suggesting that the embolization was well maintained. Because the diameter of the swelled beads is around $150 \mu \mathrm{m}$, the main hepatic artery is not embolized. It can be deduced that the cross-linked BSA beads are able to penetrate deeply and embolize the smaller and peripheral vessels, which may be related to the smooth and hydrophilic surface of the beads and their deformability; these properties provide the beads with the possibility of flowing along the vessels. However, limited data were collected to investigate the deformability of the bead in this study. BSA beads of different diameters should be investigated in the future to evaluate the deformability in the embolization of vessels of different sizes.

\section{Conclusions}

By using a water-in-oil emulsification method, BSA beads were successfully prepared and cross-linked by GA. The swelled beads have the average size of $150 \mu \mathrm{m}$, and the equilibrium swelling ratio is 2.37. Moreover, it was found that irinotecan can be easily loaded into the beads and then be slowly released from the beads. The cytotoxicity assay suggests excellent biocompatibility of the BSA beads and also proved similar antitumor activity of the irinotecan loaded BSA beads in comparison to free irinotecan. In vivo studies using a rabbit liver cancer model showed the successful embolization of liver and suggest the potential application of the drug eluting beads as chemoembolization agent for liver cancer therapy.

\section{Competing Interests}

The authors declare that they have no competing interests.

\section{Acknowledgments}

The authors acknowledge the support of the Science and Technology Support Program (Society Development) of Jiangsu Province (no. BE2012735), the National Basic Research Program of China (no. 2011CB933400), and the Foundation of Jiangsu Provincial Key Laboratory for Interventional Medical Devices (no. JR1504). The works are also a part of the project funded by the Priority Academic Program Development of Jiangsu Higher Education Institutions (PAPD).

\section{References}

[1] J. Bruix, M. Sherman, J. M. Llovet et al., "Clinical management of hepatocellular carcinoma. Conclusions of the barcelona-2000 EASL conference," Journal of Hepatology, vol. 35, no. 3, pp. 421430, 2001.
[2] A. Roche, B. V. Girish, T. de Baère et al., "Trans-catheter arterial chemoembolization as first-line treatment for hepatic metastases from endocrine tumors," European Radiology, vol. 13, no. 1, pp. 136-140, 2003.

[3] K. Stuart, "Chemoembolization in the management of liver tumors," The Oncologist, vol. 8, no. 5, pp. 425-437, 2003.

[4] J.-L. Raoul, E. Boucher, Y. Roland, and E. Garin, "131-iodine Lipiodol therapy in hepatocellular carcinoma," Quarterly Journal of Nuclear Medicine and Molecular Imaging, vol. 53, no. 3, pp. 348355, 2009.

[5] H. Ahmadzadehfar, A. Sabet, K. Wilhelm, H. J. Biersack, and J. Risse, "Iodine-131-lipiodol therapy in hepatic tumours," Methods, vol. 55, no. 3, pp. 246-252, 2011.

[6] H. T. Abada and J. Golzarian, "Gelatine sponge particles: handling characteristics for endovascular use," Techniques in Vascular and Interventional Radiology, vol. 10, no. 4, pp. 257-260, 2007.

[7] S. Imaoka, Y. Sasaki, T. Shibata et al., "A preoperative chemoembolization therapy using lipiodol, cisplatin and gelatin sponge for hepatocellular-carcinoma," Cancer Chemotherapy and Pharmacology, vol. 23, supplement 1, pp. S126-S128, 1989.

[8] L. Marelli, R. Stigliano, C. Triantos et al., "Transarterial therapy for hepatocellular carcinoma: which technique is more effective? A systematic review of cohort and randomized studies," CardioVascular and Interventional Radiology, vol. 30, no. 1, pp. 6-25, 2007

[9] H. Nishikawa, R. Kita, T. Kimura, and Y. Osaki, “Transcatheter arterial embolic therapies for hepatocellular carcinoma: a literature review," Anticancer Research, vol. 34, no. 12, pp. 6877-6886, 2014.

[10] R. Lencioni, T. De Baere, M. Burrel et al., "Transcatheter treatment of hepatocellular carcinoma with doxorubicin-loaded dc bead (DEBDOX): technical recommendations," CardioVascular and Interventional Radiology, vol. 35, no. 5, pp. 980-985, 2012.

[11] O. Jordan, A. Denys, T. De Baere, N. Boulens, and E. Doelker, "Comparative study of chemoembolization loadable beads: in vitro drug release and physical properties of DC bead and hepasphere loaded with doxorubicin and irinotecan," Journal of Vascular and Interventional Radiology, vol. 21, no. 7, pp. 10841090, 2010.

[12] K. Y. Tam, K. C.-F. Leung, and Y.-X. J. Wang, "Chemoembolization agents for cancer treatment," European Journal of Pharmaceutical Sciences, vol. 44, no. 1-2, pp. 1-10, 2011.

[13] L. Weng, H. C. Le, J. Lin, and J. Golzarian, "Doxorubicin loading and eluting characteristics of bioresorbable hydrogel microspheres: in vitro study," International Journal of Pharmaceutics, vol. 409, no. 1-2, pp. 185-193, 2011.

[14] D. J. Kerr, N. Willmott, J. H. McKillop, J. Cummings, H. J. Lewi, and C. S. McArdle, "Target organ disposition and plasma pharmacokinetics of doxorubicin incorporated into albumin microspheres after intrarenal arterial administration," Cancer, vol. 62 , no. 5 , pp. 878-883, 1988.

[15] L. Fiume, M. Baglioni, L. Bolondi, C. Farina, and G. Di Stefano, "Doxorubicin coupled to lactosaminated human albumin: a hepatocellular carcinoma targeted drug," Drug Discovery Today, vol. 13, no. 21-22, pp. 1002-1009, 2008.

[16] U. Pohlen, G. Berger, M. Binnenhei, R. Reszka, and H. J. Buhr, "Increased carboplatin concentration in liver tumors through temporary flow retardation with starch microspheres (Spherex) and gelatin powder (Gelfoam): an experimental study in liver tumor-bearing rabbits," Journal of Surgical Research, vol. 92, no. 2, pp. 165-170, 2000. 
[17] C.-J. Johansson, "Pharmacokinetic rationale for chemotherapeutic drugs combined with intra-arterial degradable starch microspheres (Spherex $\left.{ }^{\circledR}\right)$," Clinical Pharmacokinetics, vol. 31, no. 3, pp. 231-240, 1996.

[18] E. B. Denkbaş, M. Seyyal, and E. Pişkin, “5-Fluorouracil loaded chitosan microspheres for chemoembolization," Journal of Microencapsulation, vol. 16, no. 6, pp. 741-749, 1999.

[19] Y. Misirli, E. Öztürk, H. Kurşaklioğlu, and E. B. Denkbaş, "Preparation and characterization of Mitomycin-C loaded chitosan-coated alginate microspheres for chemoembolization," Journal of Microencapsulation, vol. 22, no. 2, pp. 167-178, 2005.

[20] J. S. Kim, B. K. Kwak, H. J. Shim et al., "Preparation of doxorubicin-containing chitosan microspheres for transcatheter arterial chemoembolization of hepatocellular carcinoma," Journal of Microencapsulation, vol. 24, no. 5, pp. 408-419, 2007.

[21] L. Weng, P. Rostamzadeh, N. Nooryshokry, H. C. Le, and J. Golzarian, "In vitro and in vivo evaluation of biodegradable embolic microspheres with tunable anticancer drug release," Acta Biomaterialia, vol. 9, no. 6, pp. 6823-6833, 2013.

[22] R. Silva, H. Ferreira, A. C. Carvalho, A. C. Gomes, and A. Cavaco-Paulo, "Protein microspheres as suitable devices for piroxicam release," Colloids and Surfaces B: Biointerfaces, vol. 92, pp. 277-285, 2012.

[23] C. Weber, C. Coester, J. Kreuter, and K. Langer, "Desolvation process and surface characterisation of protein nanoparticles," International Journal of Pharmaceutics, vol. 194, no. 1, pp. 91-102, 2000.

[24] B. G. Müller, H. Leuenberger, and T. Kissel, "Albumin nanospheres as carriers for passive drug targeting: an optimized manufacturing technique," Pharmaceutical Research, vol. 13, no. 1, pp. 32-37, 1996.

[25] F. Galisteo-González and J. A. Molina-Bolívar, "Systematic study on the preparation of BSA nanoparticles," Colloids and Surfaces B: Biointerfaces, vol. 123, pp. 286-292, 2014.

[26] K. I. Saleh, M. A. Ibrahim, and T. M. Faris, "Preparation and evaluation of theophylline loaded bovine serum albumin microspheres," Bulletin of Pharmaceutical Sciences, vol. 32, no. 1, pp. 65-84, 2009.

[27] T. Tanaka, H. Nishiofuku, Y. Hukuoka et al., "Pharmacokinetics and antitumor efficacy of chemoembolization using $40 \mu \mathrm{m}$ irinotecan-loaded microspheres in a rabbit liver tumor model," Journal of Vascular and Interventional Radiology, vol. 25, no. 7, pp. 1037-1044.e2, 2014.

[28] R. Neijzen, M. Q. Wong, N. Gill et al., "Irinophore $C^{\mathrm{TM}}$, a lipid nanoparticulate formulation of irinotecan, improves vascular function, increases the delivery of sequentially administered 5FU in HT-29 tumors, and controls tumor growth in patient derived xenografts of colon cancer," Journal of Controlled Release, vol. 199, pp. 72-83, 2015.

[29] A. Simionescu, D. Simionescu, and R. Deac, "Lysine-enhanced glutaraldehyde crosslinking of collagenous biomaterials," Journal of Biomedical Materials Research, vol. 25, no. 12, pp. 14951505, 1991. 

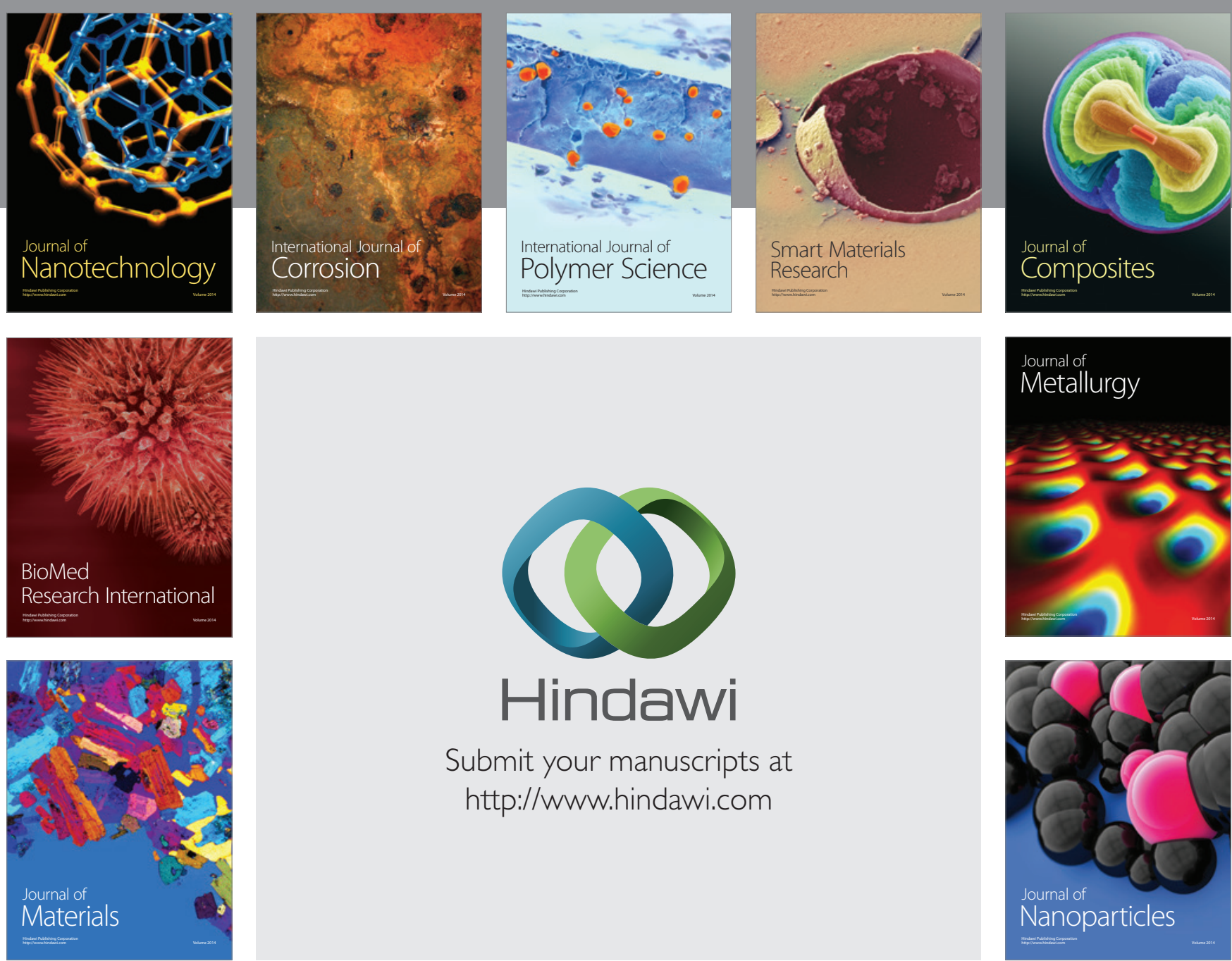

\section{Hindawi}

Submit your manuscripts at

http://www.hindawi.com

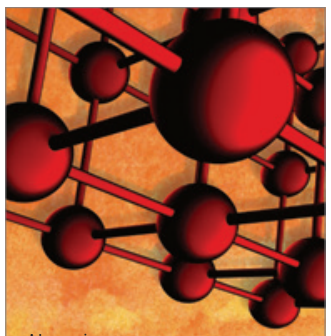

Materials Science and Engineering
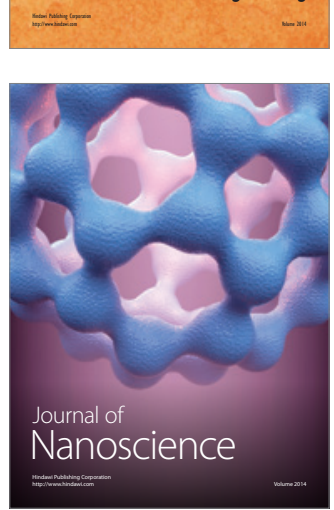
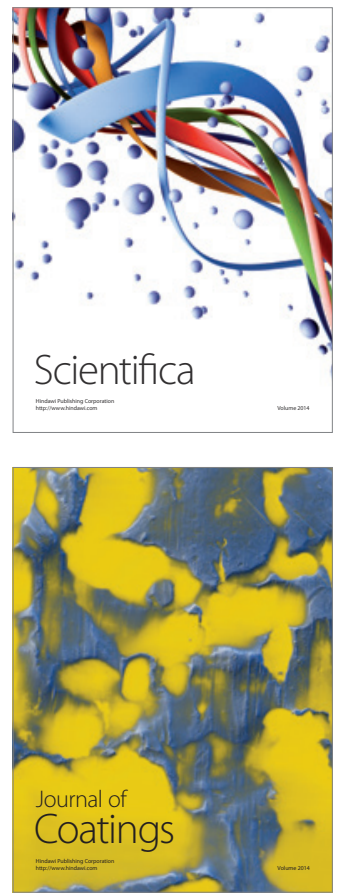
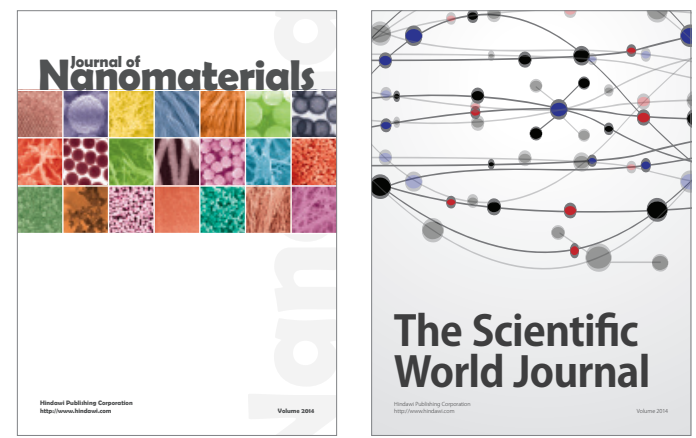

The Scientific World Journal
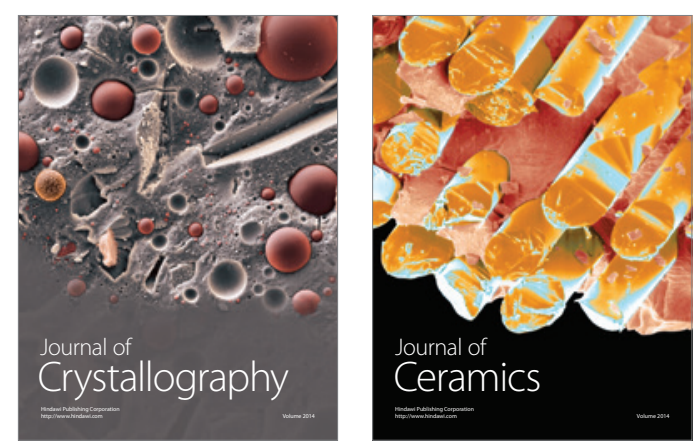
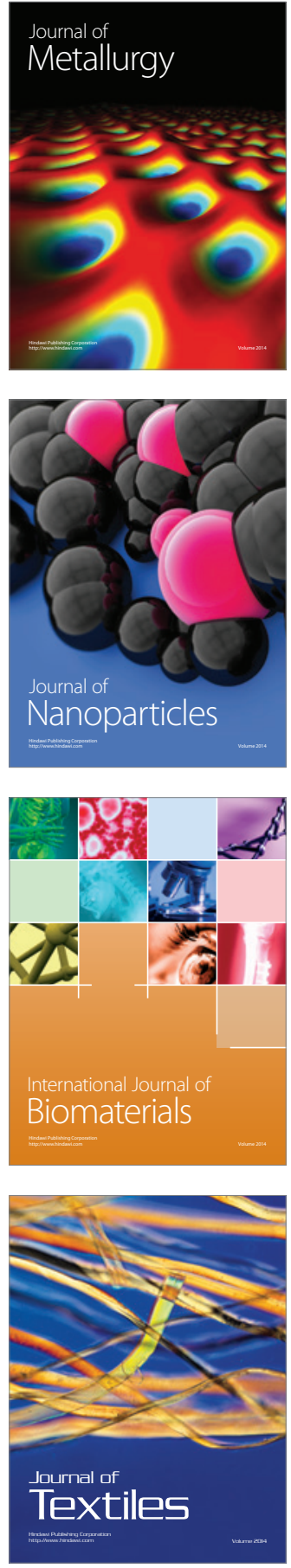